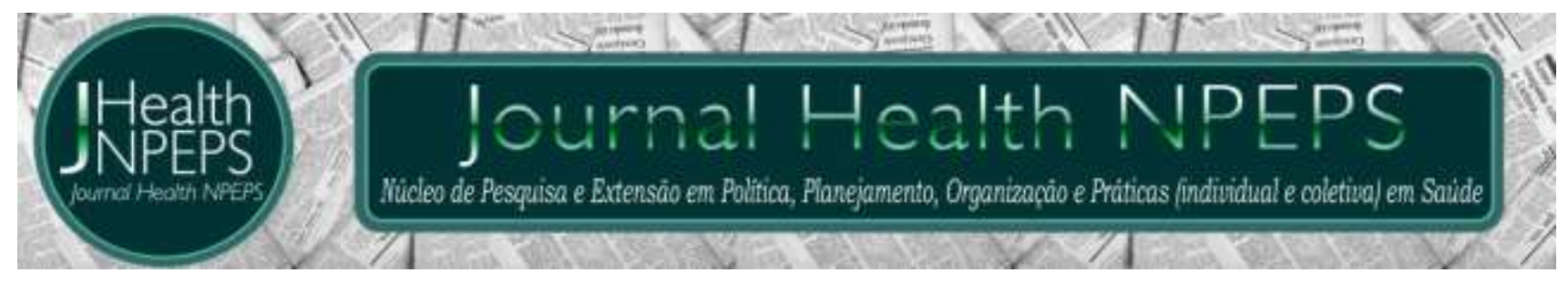

http://dx.doi.org/10.30681/252610104535

ARTIGO ORIGINAL

\title{
Região Norte do Brasil e a pandemia de COVID-19: análise socioeconômica e epidemiológica
}

\section{North region of Brazil and the COVID-19 pandemic: socioeconomic and epidemiologic analysis}

\section{Región Norte de Brasil y la pandemia de COVID-19: análisis socioeconómico y epidemiológico}

\author{
Flávia Daspett Mendonça ${ }^{1}$, Santiago Soares Rocha², Daniel Lucas Pimenta Pinheiro ${ }^{3}$, \\ Stefan Vilges de Oliveira ${ }^{4}$
}

\section{RESUMO}

Objetivo: analisar o perfil epidemiológico das doenças respiratórias e os indicadores socioeconômicos e de assistência em saúde da região Norte do Brasil durante a pandemia de COVID-19. Método: estudo epidemiológico, de caráter descritivo e quantitativo, em relação ao período de janeiro de 2010 a fevereiro de 2020. Os dados foram coletados no DATASUS e no boletim epidemiológico sobre a COVID-19 do Ministério da Saúde. Os resultados foram dispostos em números absolutos, frequência relativa e medidas de tendência central. Resultados: no período houve 1.163.303 internações, consumindo 891.494.215,40 reais. Pará e Amazonas somam 66\% desses gastos. Os meses de abril, maio e junho apresentaram médias maiores de hospitalizações durante todos os anos. A faixa etária mais onerosa foi entre 60 e 69 anos. A região Norte é menos provida de médicos, leitos de UTI e respiradores. Amazonas, Amapá e Roraima têm os maiores coeficientes de incidência de COVID-19 por 1.000 .000 de habitantes. Conclusão: a pandemia aumenta a vulnerabilidade socioeconômica e assistencial do sistema de saúde do Norte brasileiro, com sobrecarga e número de óbitos crescente. Portanto, há necessidade urgente de realocar recursos e reorganizar a rede de atenção à saúde.

\footnotetext{
${ }^{1}$ Acadêmica do curso de Medicina da Universidade Federal de Uberlândia (UFU). Uberlândia, Minas Gerais, Brasil. Email: flaviadaspett@ufu.br ORCID ID: https://orcid.org/0000-0002-2691-6100

${ }^{2}$ Acadêmico do curso de Medicina da Universidade Federal de Uberlândia (UFU). Uberlândia, Minas Gerais, Brasil. Email: sansoares25@gmail.com ORCID ID: https://orcid.org/0000-0001-8570-8224

${ }^{3}$ Acadêmico do curso de Medicina da Universidade Federal de Uberlândia (UFU). Uberlândia, Minas Gerais, Brasil. Email: danielpinm@gmail.com ORCID ID: https://orcid.org/0000-0001-9436-8479

${ }^{4}$ Biólogo. Doutor em Medicina Tropical. Professor Adjunto do Departamento de Saúde Coletiva, Faculdade de Medicina, Universidade Federal de Uberlândia (UFU). Uberlândia, Minas Gerais, Brasil. E-mail: stefan@ufu.br ORCID ID: https://orcid.org/0000-0002-5493-2765 Autor principal - Endereço para correspondência: Departamento de Saúde Coletiva. Avenida Pará, 1720, Campus Umuarama, Bloco 2U, Sala 8, Umuarama, CEP 38405320. Uberlândia, Minas Gerais, Brasil.
}

Este artigo está licenciado sob forma de uma licença Creative Commons Atribuição 4.0 Internacional, que permite uso irrestrito, distribuição e reprodução em qualquer meio, desde que a publicação original seja corretamente citada. 
Descritores: Infecções por Coronavirus; Análise Socioeconômica; Pesquisa sobre Serviços de Saúde; Epidemiologia Descritiva; Brasil.

\begin{abstract}
Objective: to analyze the epidemiological profile of respiratory diseases and the socioeconomic and health care indicators of northern Brazil during the COVID-19 pandemic. Method: epidemiological study, of a descriptive and quantitative character, in relation to the period from January 2010 to February 2020. Data were collected in DATASUS and in the epidemiological bulletin on COVID-19 of the Ministry of Health. The results were displayed in absolute numbers, relative frequency and measures of central tendency. Results: in the period there were 1,163,303 hospitalizations, consuming $891,494,215.40$ reais. Pará and Amazonas account for $66 \%$ of these expenses. The months of April, May and June showed higher averages of hospitalizations during all years. The most expensive age group was between 60 and 69 years. The northern region is less equipped with doctors, ICU beds and respirators. Amazonas, Amapá and Roraima have the highest incidence coefficients of COVID-19 per 1,000,000 inhabitants. Conclusion: the pandemic increases the socioeconomic and assistance vulnerability of the health system in northern Brazil, with an overload and an increasing number of deaths. Therefore, there is an urgent need to reallocate resources and reorganize the health care network.
\end{abstract}

Descriptors: Coronavirus Infections; Socioeconomic Analysis; Health Services Research; Epidemiology, Descriptive; Brazil.

\title{
RESUMEN
}

Objetivo: analizar el perfil epidemiológi co de las enfermedades respiratorias y los indicadores socioeconómicos y de salud del Norte de Brasil durante la pandemia COVID19. Método: estudio epidemiológico, de carácter descriptivo y cuantitativo, en relación con el período comprendido entre enero de 2010 y febrero de 2020. Los datos se recopilaron en DATASUS y en el boletín epidemiológico sobre COVID-19 del Ministerio de Salud. Los resultados se mostraron en números absolutos. frecuencia relativa y medidas de tendencia central. Resultados: en el período hubo 1.163.303 hospitalizaciones, que consumieron $891.494 .215,40$ reales. Pará y Amazonas representan el $66 \%$ de estos gastos. Los meses de abril, mayo y junio mostraron promedios más altos de hospitalizaciones durante todos los años. El grupo de edad más caro fue entre 60 y 69 años. La región Norte está menos equipada con médicos, camas de $\mathrm{UCl}$ y respiradores. Amazonas, Amapá y Roraima tienen las tasas más altas de incidencia de COVID-19 por cada 1,000,000 de habitantes. Conclusión: la pandemia aumenta la vulnerabilidad socioeconómica y asistencial del sistema de salud en el Norte de Brasil, con una sobrecarga y un número creciente de muertes. Por lo tanto, existe una necesidad urgente de reasignar recursos y reorganizar la red de atención médica.

Descriptores: Infecciones por Coronavirus; Análisis Socioeconómico; Investigación sobre Servicios de Salud; Epidemiología Descriptiva; Brasil.

\section{INTRODUÇÃO}

0 pulmão é o órgão mais vulnerável às infecções do ambiente externo, devido à constante exposição à partículas, produtos químicos e organismos infecciosos. Por consequência, quatro das dez causas 
mais comuns de morte no planeta são decorrentes de doenças do trato respiratório ${ }^{1}$. Estima-se que 65 milhões de pessoas sofrem da doença pulmonar obstrutiva crônica (DPOC) $)^{2}$ e que, durante um ano, ela causa cerca de 3 milhões de óbitos no mundo, o que a torna a terceira principal causa de morte a nível global ${ }^{3}$.

As infecções das vias aéreas inferiores, por sua vez, ocupam o quarto lugar nesse ranking ${ }^{3}$. Elas compreendem a bronquite aguda, as bronquiolites, as infecções em pacientes com bronquiectasias e as infecções que comprometem os espaços aéreos, como as pneumonias ${ }^{4}$. Já as neoplasias do trato respiratório são a sexta mais comum causa de óbito, com aproximadamente 1,7 milhão de mortes em um ano, no mundo ${ }^{3}$. A tuberculose, a décima causa mais comum, ocasiona a morte de quase 1,3 milhão de pessoas anualmente no mundo ${ }^{3}$.

Do ponto de vista de morbidade, o impacto das doenças respiratórias na população pode ser mensurado por meio dos anos de vida perdidos por morte ou incapacidade (DALYs - disability-adjusted (ife-years). Assim, um DALY significa um ano perdido de vida saudável ${ }^{5}$. Nesse sentido, as afecções do trato respiratório representam mais de $10 \%$ de todos os
DALYs, atrás apenas das enfermidades cardiovasculares ${ }^{1}$.

Embora as doenças respiratórias levem à incapacidade e à morte em todas as regiões e classes sociais do mundo. A pobreza, a aglomeração, as exposições ambientais e, em geral, as más condições de vida suscetibilizam os indivíduos a esse grupo de enfermidades $^{1}$. Nessa perspectica, o Brasil, é extensamente afetado, uma vez que, em 2018, 13,5 milhões de brasileiros viviam com até U\$ 2 por dia, em situação de extrema pobreza, contingente considerado recorde em sete anos de análise. No mesmo ano, um quarto da população brasileira, 52,5 milhões de pessoas, vivia com menos de R\$ 420 per capita por mês 6 . Sabe-se que, no país, as infecções de vias aéreas inferiores e a DPOC constituem a terceira e a quinta principal causa de morte, respectivamente ${ }^{7}$.

O contexto da região Norte do país é ainda mais alarmante, posto que todos os seus estados apresentam indicadores de pobreza acima da média nacional ${ }^{6}$. Essa situação representa a discrepância socioeconômica entre as regiões administrativas do Brasil, cuja grandeza territorial traduz uma nação multifacetada pela desigualdade. Por conseguinte, os impactos das doenças 
respiratórias no Sistema Único de Saúde (SUS) agravar-se-ão nesse perfil de região durante a pandemia da COVID-19, ao demonstrar fragilidades quanto ao acesso, acessibilidade e assistência em saúde ${ }^{6}$.

Foi observado até o dia 06 de maio de 2020, que foram registrados 116.243 casos confirmados da COVID-19 e 8.014 óbitos decorrentes dessa enfermidade no Brasil, além de um coeficiente de letalidade de $6,9 \%^{8}$. A região Norte apresenta aproximadamente $22 \%$ do total dos casos. 0 estado do Amazonas, por sua vez, tem 12 das 20 cidades de maior incidência de casos do novo coronavírus no Brasil e cinco cidades entre as $10 \mathrm{com}$ maior coeficiente de letalidade, com o município de Manacapuru (AM) em primeiro lugar no país?.

Por isso, é imprescindível a análise atualizada das internações hospitalares por doenças respiratórias no Norte brasileiro, bem como da disponibilidade de recursos em saúde para o enfrentamento dessas e das subsequentes, decorrentes das infecções como, por exemplo, pela COVID-19. Dessa forma, o objetivo deste estudo é analisar o perfil epidemiológico das doenças respiratórias e os indicadores socioeconômicos e de assistência em saúde da região Norte do Brasil durante a pandemia da COVID-19.

\section{MÉTODO}

Estudo epidemiológico, de caráter descritivo e quantitativo, acerca do número de internações e de óbitos por afecções respiratórias ocorridos na região Norte do Brasil, no período de $1^{\circ}$ de janeiro de 2010 a 29 de fevereiro de 2020, e de seus recursos de saúde, em fevereiro de 2020. Foram consideradas afecções respiratórias as patologias do Capítulo $X$ da Classificação Estatística Internacional de Doenças e Problemas Relacionados com a Saúde (CID-10), que abrange as infecções agudas do trato respiratório superior (faringite, amigdalite, laringite, traqueíte $\mathrm{e}$ outras), inferior (bronquite $\mathrm{e}$ bronquiolite aguda, pneumonia, Influenza), doenças crônicas das vias aéreas superiores e das vias aéreas inferiores, e outras doenças do aparelho respiratório.

A região Norte compreende sete estados: Acre, Amazonas, Amapá, Pará, Rondônia, Roraima e Tocantins. Juntos, eles abrangem área territorial de $3.851 .281 \mathrm{~km}^{210}$, representando quase 50\% do território nacional. Em 2019, a população estimada para a região era de 
18.430.980 habitantes ${ }^{11}$, com densidade demográfica de 4,79 hab/km ${ }^{29}$. Conforme o Censo Demográfico de 201012, a média dos Índices de Desenvolvimento Humano (IDH) dos estados que a compõem a região é de 0,683 .

No estudo foram utilizados dados provenientes do Sistema de Informação Hospitalar do Sistema Único de Saúde (SIH/SUS), disponíveis no endereço eletrônico do Departamento de Informática do SUS (DATASUS) ${ }^{13}$ sobre as seguintes variáveis: número de internações e de óbitos por causas respiratórias, taxa de mortalidade, faixa etária em anos categorizados (menor de 1 ano; 1 a 4; 5 a 9; 10 a 14; 15 a 19; 20 a 29; 30 a 39; 40 a 49; 50 a 59; 60 a 69; 70 a 79; e 80 ou mais), valor total dos gastos, valor médio de internação e média de permanência, mês e ano de processamento, concernentes ao período de 2010 a 2019 e os dois primeiros meses de 2020.

As informações foram colhidas das respectivas fontes de dados no dia 18 de abril de 2020 e, posteriormente, o programa Microsoft Excel, versão 2016, foi utilizado para realização dos cálculos e construção de gráficos e de tabelas. A partir de uma análise estatística descritiva, os dados são apresentados em números absolutos, em frequência relativa e em medidas de tendência central. Assim, para determinar a variação percentual do número de internações na região Norte e no Brasil, foram colhidos os números de internações em cada ano por região e no Brasil. Para o cálculo da taxa média de internações por 100 mil habitantes, foi realizada a divisão do número de internações de cada ano (2010 a 2019), pela respectiva população estimada ${ }^{10} \mathrm{e}$ a multiplicação do resultado por 100.000 . Em seguida, foi calculada a média aritmética dos valores. Posteriormente, a variação percentual da taxa de mortalidade foi calculada para cada ano utilizando a taxa de 2010 como base.

A análise do número de internações e óbitos por mês na região Norte, de janeiro de 2010 a fevereiro de 2020, se deu da seguinte forma: calculou-se a média aritmética do número de internações por causas respiratórias do mês de janeiro de cada um dos anos, do mês de fevereiro de cada um, e assim por diante, levando em conta o fato de que a partir de março há apenas 10 meses sendo analisados, ou seja, a divisão a ser feita na média é por 10. As mesmas operações foram realizadas com o número de óbitos 
causados por afecções do capítulo $X$ do CID-10.

Para avaliação do Cadastro Nacional de Estabelecimentos de Saúde

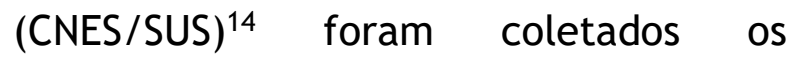
seguintes dados, de cada região e do país: número de leitos de UTI (Unidade de Tratamento Intensivo) adulto I, adulto II e adulto III, número de profissionais médicos e número de respiradores em uso. A taxa dessas variáveis por 100 mil habitantes foi obtida a partir da divisão do respectivo valor pela população estimada em 2019 de cada região ${ }^{10}$, seguida da multiplicação desses valores por 100.000. Além disso, o Instituto Brasileiro de Geografia e Estatística $(I B G E)^{11}$ foi a fonte de pesquisa para 0 índice de Desenvolvimento Humano (IDH) e para a renda média mensal dos estados brasileiros. A partir do boletim epidemiológico do Ministério da Saúde $(M S)^{15}$ foram retirados os coeficientes de incidência e mortalidade por COVID-19 por 1.000 .000 de habitantes relativos aos estados e às capitais brasileiras.

O estudou respeitou todos os aspectos éticos em pesquisa, mas pela natureza do estudo, baseado em dados secundários, de domínio público, não houve a submissão ao Comitê de Ética em Pesquisa (CEP) de acordo com a
Resolução do Conselho Nacional de Saúde $\mathrm{n}^{\circ} 466$, de 12 de dezembro de $2012^{16}$.

\section{RESULTADOS}

Entre janeiro de 2010 e fevereiro de 2020, 1.163.303 pessoas foram internadas nas unidades do Sistema Único de Saúde (SUS) na região Norte, devido a doenças respiratórias. Nos estados do Pará, Amazonas, Rondônia e Tocantins têm-se a maior parte das internações, com 648.019, 167.936, 136.670 e 96.649 entradas de pacientes no SUS, respectivamente. Acre, Amapá e Roraima apresentaram 41.460, 39.884 e 32.685 hospitalizações, nessa ordem. Percebe-se que houve um decréscimo no número de pessoas internadas no SUS, ao comparar 2010 com 137.427 internações, e 2019 com 103.711. Entretanto, tem-se números de internações semelhantes em 2017 e 2018, com um incremento de aproximadamente $10 \%$ em relação a 2016. No período de 2010 a 2019, ocorreram 675 internações por 100.000 habitantes na região Norte. As regiões, Sul, Centro-Oeste, Nordeste e Sudeste apresentaram 920, 672, 600 e 535 hospitalizações por 100.000 habitantes, respectivamente. Por fim, destacam-se números médios maiores de internações 
e de óbitos nos meses de abril a junho

deste período de 11 anos (Figura 1).

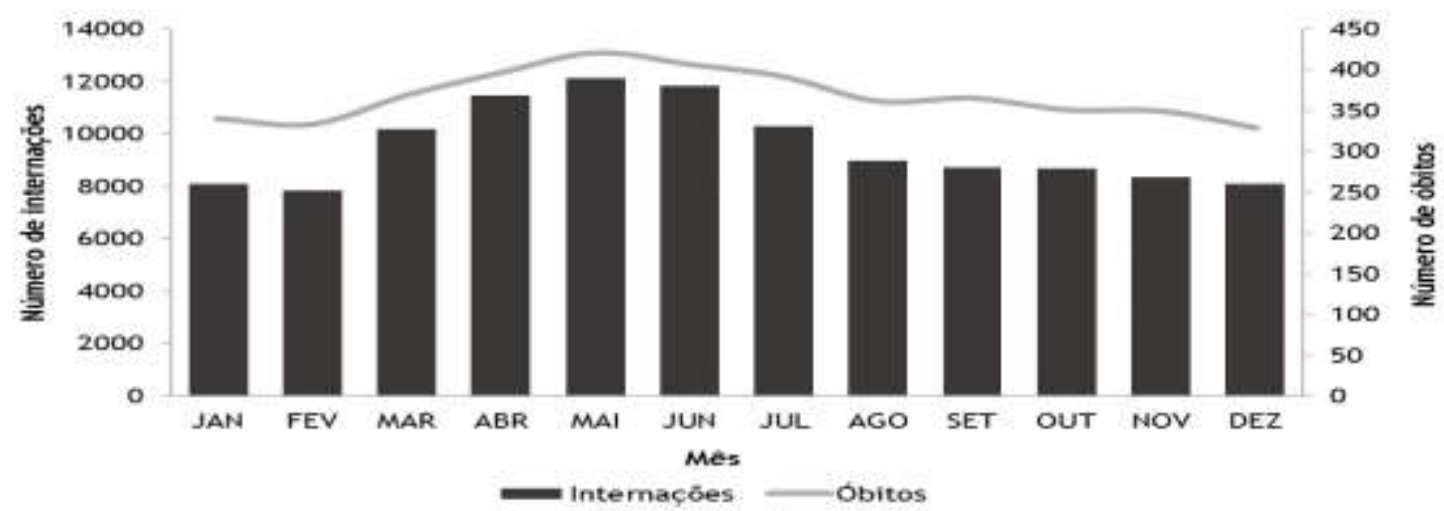

Figura 1 - Média de internações e óbitos por causas respiratórias, por meses do ano, na região Norte, de janeiro de 2010 a fevereiro de 2020.

As hospitalizações ocasionadas por doenças respiratórias, entre os anos de 2010 e 2020, custaram 891.494.215,40 ao SUS em toda a região Norte. É possível observar que o estado do Pará é o maior contribuinte desse valor, pois suas internações custaram um total de $435.792 .450,40$ reais, seguido pelo estado do Amazonas (156.853.106,70 reais). Nesse cenário, evidencia-se também que, durante esse período, o valor médio de cada internação foi de 766,35 reais no Norte do país.
Entre os estados de Roraima, Amazonas, Tocantins e Acre apresentaram os maiores valores médios de internação, sendo 1.040, 934, 881 e 854 reais, respectivamente. Seguindo a mesma perspectiva, nas demais regiões, Sudeste $(1.093,76)$, Sul $(1.032,2)$, Centro-Oeste $(965,7)$ e Nordeste $(892,37)$, encontram-se maiores valores médios por hospitalização. Por outra perspectiva, de acordo com as Tabelas 1, 2 e 3, nota-se que a região Norte possui números menores de leitos de UTI, médicos e respiradores.

Tabela 1 - Número de leitos de UTI e número de leitos de UTI por 100.000 habitantes por região, em fevereiro de 2020.

\begin{tabular}{lcc}
\hline \multicolumn{1}{c}{ Região } & Leitos de UTI & $\begin{array}{c}\text { Leitos de UTI por 100.000 } \\
\text { habitantes }\end{array}$ \\
\hline Norte & 1355 & 7,35 \\
Nordeste & 5912 & 10,36 \\
Centro-Oeste & 2914 & 17,88 \\
Sudeste & 16114 & 18,23 \\
Sul & 4479 & 14,94 \\
\hline
\end{tabular}


Tabela 2 - Número de médicos e número de médicos por 100.000 habitantes por região, em fevereiro de 2020.

\begin{tabular}{lcc}
\hline \multicolumn{1}{c}{ Região } & $\begin{array}{c}\text { Número de } \\
\text { médicos }\end{array}$ & $\begin{array}{c}\text { Número de médicos por } \\
\mathbf{1 0 0 . 0 0 0} \text { habitantes }\end{array}$ \\
\hline Norte & 19430 & 105,42 \\
Nordeste & 76076 & 133,30 \\
Centro-Oeste & 32938 & 202,11 \\
Sudeste & 215765 & 244,16 \\
Sul & 68161 & 227,39 \\
\hline
\end{tabular}

Tabela 3 - Número de respiradores e número de respiradores por 100.000 habitantes por região, em fevereiro de 2020.

\begin{tabular}{lcc}
\hline \multicolumn{1}{c}{ Região } & $\begin{array}{c}\text { Número de } \\
\text { respiradores }\end{array}$ & $\begin{array}{c}\text { Número de respiradores por } \\
\mathbf{1 0 0 . 0 0 0} \text { habitantes }\end{array}$ \\
\hline Norte & 3521 & 19,10 \\
Nordeste & 12528 & 21,95 \\
Centro-Oeste & 6168 & 37,85 \\
Sudeste & 33806 & 38,25 \\
Sul & 9388 & 31,32 \\
\hline
\end{tabular}

Quanto ao custo com serviços hospitalares por faixa etária, o valor tende a crescer com a idade. Em todas as regiões do Brasil, é possível observar que os idosos com mais de 50 anos são os mais onerosos. Na região Norte, a faixa mais dispendiosa é a que compreende dos 60 aos 69 anos, com custos médios de 1039,56 reais por internação. Por fim, nota-se que Amazonas e Roraima são os estados que apresentam os maiores valores médios de internação por período etário.

A média de permanência por faixa etária de pessoas internadas em unidades do SUS na região Norte é de 4,4 dias. Assim, observa-se uma média de 6,8 dias em crianças com menos de 1 ano, por outro lado, no período etário de 1 a 49 anos, tem-se uma variação de 4 a 5,1 dias de permanência. Já a média de estadia no grupo de pessoas com 50 anos ou mais é de 6 a 6,3 dias. Por fim, ressalta-se que, nessa região, crianças com menos de 1 ano geram um gasto médio de 742,81 reais por internação e, em idosos com mais de 50 anos, esse valor ultrapassa 934,44 reais.

Entre 2010 e 2014, a taxa de mortalidade, vinculada a doenças do aparelho respiratório, variou de 2,44 a $3,62 \%$ na região Norte. Com isso, é possível observar um aumento gradativo desses números que foram de 4,18\% em 2015 a 5,17\% em 2019. Destaca-se que até fevereiro de 2020, a taxa de 
mortalidade evidenciada nesse ano era de $6,55 \%$. Por outro lado, no Brasil, esse parâmetro variou de 5,4 a 7,53\%, entre 2010 e 2015. Nota-se um aumento considerável dessa taxa em 2016 (8,24\%) e, até fevereiro de 2020, observa-se um incremento ainda maior, alcançando 9,96\%. Os períodos entre agosto e janeiro desses 11 anos de dados acumulados, na região Norte, apresentam os maiores parâmetros de mortalidade. Assim, evidenciando taxas mais elevadas, entre 5 e 6,6\%, destacam-se 2018, 2019 e os dois meses iniciais de 2020.

Por último, observa-se que houve crescimento da taxa de mortalidade das faixas etárias de 60 a 69 anos (Figura 2 A), 70 a 79 anos (Figura 2 B) e 80 anos ou mais (Figura 2 C) em relação a 2010, a nível nacional e, principalmente, na região Norte, com destaque para a faixa de 60 a 69 anos, a qual aumentou 113,15\% (Figura 2 A).
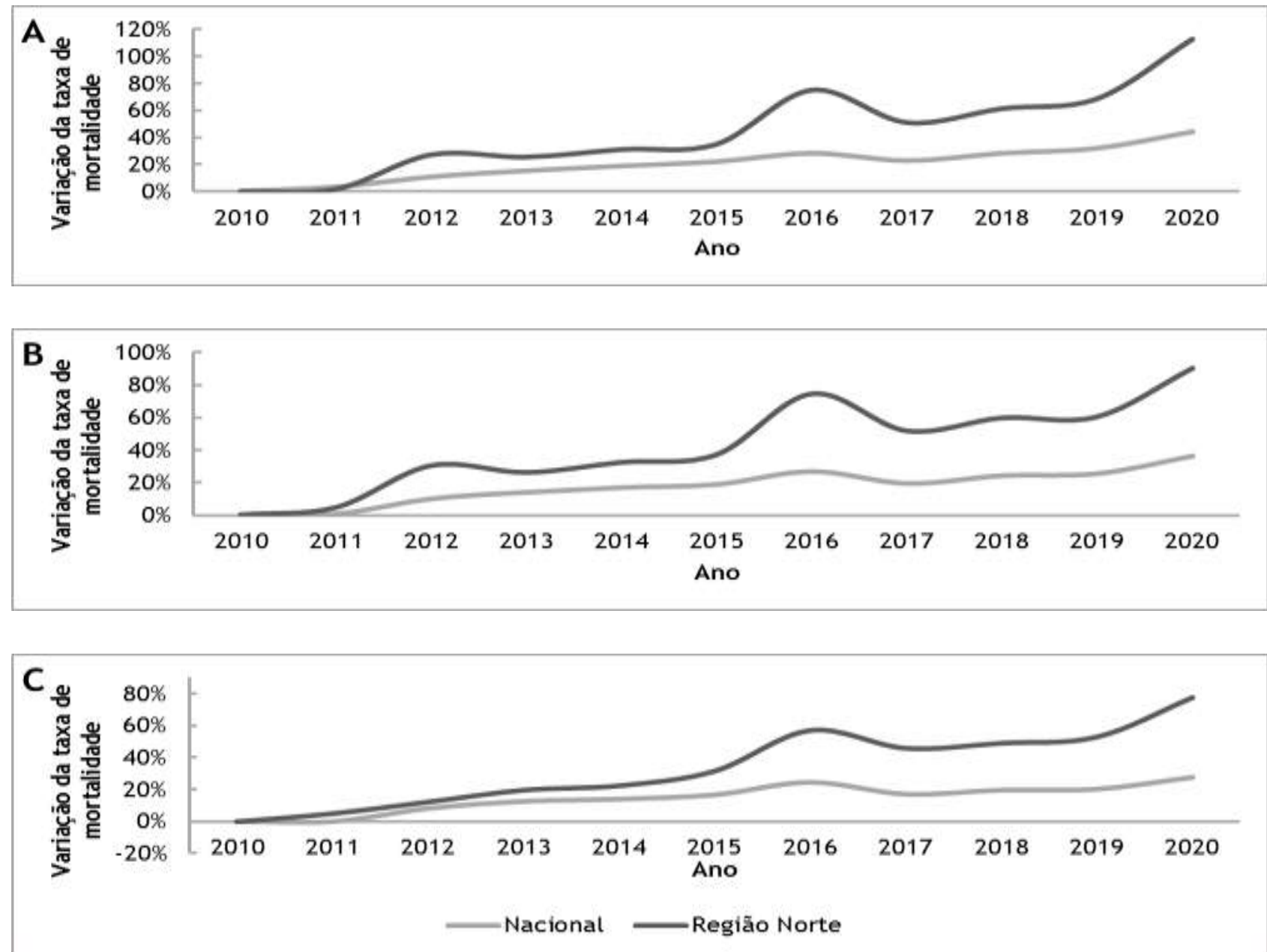

Figura 2 - Variação do percentual da taxa de mortalidade (segundo o ano base de 2010) por doenças respiratórias da Região Norte e do Brasil de 2010 a 2020, de acordo com as faixas etárias: A - 60 a 69 anos, B - 70 a 79 anos, C - 80 anos ou mais. 
No período total analisado observa-se que a maior parte dos óbitos ocorrem entre os acima de 60 anos, totalizando 29.283 mortes, o que corresponde a mais de $65 \%$ do total de mortes da região Norte. Os idosos com 80 anos ou mais compreendem a faixa etária com mais óbitos (12.557). Quanto aos estados, destacam-se Pará, Amazonas e Rondônia com 19.297, 7.655 e 6.021 falecimentos por doenças respiratórias, respectivamente. Tocantins, Acre, Roraima e Amapá apresentam 4.992, 3.228, 1.910 e 1.735 óbitos, nessa ordem.

A taxa de mortalidade média da região Norte, no período de 2010 a fevereiro de 2020, é de 1,24\% em crianças menores de 1 ano, e, a partir dos 10 anos de idade, progride crescentemente ao decorrer dos anos de vida, atingindo 16,68\% em idosos com 80 anos ou mais. Assim, nessa última faixa etária, Amapá (25,42\%), Acre (25,25\%), Roraima (23,02\%) e Amazonas (19,44\%) apresentam os maiores parâmetros de mortalidade média. Em contrapartida, no Brasil, essa taxa não ultrapassa 1\% até a faixa etária de 14 anos e, em idosos com 80 anos ou mais, alcança $21,77 \%$.
Em outra perspectiva, em 2019, a renda mensal domiciliar per capita média da região Norte foi 950,71 reais, superior apenas à região Nordeste, que apresentou um valor de 889,22 reais. Por conseguinte, Sul (1744,33 reais), CentroOeste $(1727,25$ reais), Sudeste $(1665,75)$ evidenciaram os maiores parâmetros mencionados. Dentre os estados do Norte do país, Amazonas (842 reais) e Pará (807 reais) apresentam os menores valores de renda. De outro modo, ressalta-se que nenhum estado dessa região figurou entre os dez melhores IDHs do país em 2010. Foi observado que o estado do Amapá $(0,708)$ é a unidade federativa que apresenta o melhor IDH da região Norte, em $12^{\circ}$ lugar; Já Amazonas $(0,674)$, Acre $(0,663)$ e Pará $(0,646)$ ocupam a $18^{\circ}, 21^{\circ}$ e $24^{\circ}$ posição, respectivamente ${ }^{12}$.

Quanto ao contexto pandêmico no país, a partir de dados nacionais ${ }^{15}$, evidencia-se que os estados com os maiores coeficientes de incidência da COVID-19 por 1.000.000 de habitantes, calculado a partir da projeção do IBGE para 2020, foram Amazonas (521), Amapá (512), Roraima (403), Ceará (381) e São Paulo (318). Nesse contexto, Manaus (762) ocupa a $5^{\circ}$ posição entre as capitais que apresentaram os maiores 
coeficientes de incidência por 1.000.000 de habitantes, ficando atrás de Fortaleza (1.007), São Luís (899), Recife (850) e São Paulo (789).

No coeficiente de mortalidade pela COVID-19 por 1.000.000 de habitantes dos estados brasileiros, também embasado na projeção do IBGE para 2020, é possível observar que os maiores resultados foram registrados por Amazonas (45), Pernambuco (24), Rio de Janeiro (24), São Paulo (23) e Ceará (22). Ademais, entre as capitais, Manaus (72), Recife (61) e São Paulo (57) apresentaram os maiores coeficientes de mortalidade por essa doença.

\section{DISCUSSÃO}

Durante o período do estudo, foram registradas quase 1,2 milhão de internações por doenças respiratórias no SUS, na região Norte do país, das quais os estados do Pará e Amazonas, juntos, são responsáveis por cerca de 70\%. 0 número de pacientes internados por decorrência das enfermidades do trato respiratório cresce desde 2016. No mesmo período e devido a essas afecções, houve 12.817.699 internações em todo o Brasil ${ }^{13}$. Como se identifica, as internações por enfermidades do trato respiratório acometem grande parte dos cidadãos brasileiros ${ }^{4}$. Esse fato, aliado ao número exponencial de infecções decorrentes da pandemia de COVID-19, em 2020, tende a sobrecarregar, em especial os estados que, no Norte do país, são os que projetam maiores internações por doenças respiratórias ${ }^{9}$.

Sob esse ponto de vista, percebe-se ainda, a infortuna posição da região Norte no que tange ao número de internações por doenças respiratórias a cada 100.000 habitantes, a qual se encontra em segundo lugar, atrás somente da região Sul ${ }^{13}$. Nota-se, também, que no Norte brasileiro, os meses de abril, maio e junho somam o maior número de internações, fato que se acrescenta ao aumento dos casos de infecção pelo novo coronavírus no mês de abril na região e sua continuação e impactos nos meses de maio e junho ${ }^{17}$. Assim, a pandemia será enfrentada no período mais crítico do ano, no que diz respeito a afecções respiratórias ${ }^{4}$, tornando, tal conjunção, um inegável desafio à estrutura de saúde nesse território.

Ainda, foram gastos aproximadamente 900 milhões de reais com os serviços hospitalares e dos profissionais de saúde no enfrentamento dessas enfermidades, valor que os estados do Pará e Amazonas, juntos, são 
responsáveis por 66\%. No mesmo intervalo de tempo, no Brasil, foram dispendidos quase 13 bilhões de reais. É nítido, portanto, que as doenças respiratórias não apenas afetam significativamente a população brasileira, mas também geram enorme sobrecarga monetária ao SUS 4 .

Não obstante, as regiões brasileiras não são igualmente afetadas pelas consequências das doenças respiratórias, uma vez que a região Norte é, entre todas, a que possui, proporcionalmente, o menor número de leitos de UTI, de médicos e de respiradores, os quais são amplamente necessários no tratamento e no combate não só dessas enfermidades, mas também das complicações desencadeadas pelas infecções por COVID-1918. Isso, irrefutavelmente, torna essa região mais incapacitada e reflete, de forma clara, a existência de um sistema de saúde não único, mas multifacetado pela desigualdade, já que a distribuição de recursos em saúde não é igualitária entre as unidades federativas do Brasil.

Outrossim, no período de 2010 a 2020 foram registrados 44.838 óbitos por doenças respiratórias no Norte brasileiro e 910.016 em todo o país ${ }^{13}$. Ainda, os idosos, apesar de terem a segunda maior média de permanência, atrás apenas das crianças menores de 1 ano, os idosos são os que geram maiores gastos com a internação, têm a maior taxa de mortalidade e registram o maior número de óbitos. A idade avançada se configura como fator de risco para a infecção pela COVID-19, visto que aumenta as chances de evolução para a Síndrome Respiratória Aguda Grave (SRAG), desfecho de maior gravidade que, frequentemente, demanda cuidados em Unidade de Terapia Intensiva (UTI) e uso de ventilação mecânica, além de poder culminar em óbito ${ }^{19}$. É possível notar, também, que a taxa de mortalidade por doenças respiratórias na região Norte em relação ao ano de 2010, teve maior crescimento na região Norte do que no Brasil e aumenta desde 2015, principalmente entre os idosos.

$\mathrm{Na}$ região Norte, Amazonas é o segundo Estado, tanto com maior valor médio de internação, quanto com maior número de óbitos por doenças respiratórias. Nesse local, bem como em muitos outros no país, o processo de descentralização do SUS foi dificultado, devido, sobretudo, à desconsideração das especificidades regionais pelo Governo Federal, tais como as condições de vida da população, as peculiaridades geopolíticas/isolamento geográfico, a 
baixa densidade populacional, a pobreza e a precária infraestrutura assistencial e sanitária ${ }^{12}$. Dessa forma, existem barreiras limitantes à cobertura ampla e irrestrita dos sistemas de saúde estadual e municipal nessa localidade, tornando sua exequibilidade destoante da prevista nos instrumentos reguladores do SUS 20.

A região Norte também apresenta a segunda menor renda mensal domiciliar per capita média. 0 maior parâmetro de renda encontrado, correspondente a região Sul, sendo $83,48 \%$ superior ao do Norte brasileiro. E os estados Amazonas e Pará são os estados que apresentam os menores valores de renda. Quanto ao "ranking" de IDH das 27 unidades federativas, os estados do Norte começam a aparecer apenas a partir da $12^{\mathrm{a}}$ posição. A população do Norte do país, não possui as mesmas condições de acesso aos serviços de saúde como de outras regiões do Brasil ${ }^{21}$. Esse aspecto reforça que a distribuição dos serviços de saúde não acontece de forma equânime e com a qualidade adequada em todo o território brasileiro $^{21}$.

São diversas as variáveis que tornam a população de baixa renda mais propensa à infecção pelo novo coronavírus, tais como o uso de transporte público, o maior número de moradores por domicílio, o deficitário acesso ao saneamento básico e à saúde e a dificuldade de manter 0 isolamento social sem perda importante da renda ou do trabalho ${ }^{22}$. Além desses fatores, ressalta-se também que países de baixa e média renda contêm $69 \%$ da população global com 60 anos ou mais, situação alarmante, visto que esses indivíduos são os mais gravemente afetados por esse patógeno ${ }^{23}$. Nesse sentido, três estados do Norte já possuem a maior incidência da COVID-19, com Amazonas em $1^{\circ}$ lugar no Brasil e Manaus em $5^{\circ}$, no que tange às capitais. Ainda, no quesito mortalidade pela COVID-19, Amazonas e Manaus configuram-se em $1^{\circ}$ lugar $^{15}$.

Por isso, a prevenção das doenças respiratórias aumentará significativamente a disponibilidade de leitos, além de diminuir, de forma considerável, o dispêndio do SUS. Tal fato, somado à ampliação da rede hospitalar, ao redirecionamento e à reorganização de serviços, contribuirá para amenizar a situação caótica provocada pela pandemia da COVID-1924. Nessa perspectiva, intervenções governamentais preventivas, como detecção precoce, uso de máscaras, quarentena oportuna e melhor estratégia terapêutica, reduzem a probabilidade de infecção por exposição e diminuem o 
tempo de recuperação. Por conseguinte, evidencia-se a necessidade de intervenções contínuas e de vigilância, o que é possível com aportes financeiros maiores provenientes do governo ${ }^{25}$.

Por fim, se coloca em voga o princípio da equidade, um dos pilares finalísticos do SUS e, atualmente, o tema central das discussões sobre as reformas dos sistemas de saúde no mundo ocidental. Esse fundamento se refere à necessidade de se "tratar desigualmente os desiguais" a fim de se garantir a igualdade de oportunidades de sobrevivência e de desenvolvimento pessoal e social entre os indivíduos ${ }^{21}$. Com esse fito, é imperioso reconhecer a desigualdade entre as pessoas e os grupos sociais, bem como sua natureza injusta e a necessidade de superá-las ${ }^{21}$.

Entre os empecilhos desse estudo, ressaltam-se limitações vinculadas à própria base de dados do DATASUS, visto que existem ocasionais inconformidades de dados fornecidos pelos profissionais de saúde. Nesse cenário, evidencia-se a necessidade de discutir métodos para melhorar o preenchimento dos formulários de notificação, a fim de torná-los completos e menos suscetíveis a erros. Além disso, salienta-se a relevância de futuros estudos para disponibilizar informações atualizadas a respeito do perfil epidemiológico da COVID-19 a nível regional, com o intuito de direcionar a assistência a gestores e a profissionais de saúde nas ações de combate desse patógeno de acordo com as particularidades inerentes a cada região.

\section{CONCLUSÃO}

À luz do que foi abordado, observa-se que foram gastos quase 1 bilhão de reais na assistência a cidadãos acometidos, sendo que mais da metade desse valor advém do Pará e do Amazonas. 0 número de internações e a taxa de mortalidade aumentaram desde 2010, principalmente na faixa etária acima de 60 anos. Além de a região ser possuidora da segunda menor renda mensal per capita, os IDH de cada estado também são menores, sendo o mais alto ocupante da $12^{\mathrm{a}}$ posição. Com relação a média mensal de internações e mortes, os meses de abril, maio e junho caracterizam o período mais crítico.

A região Norte apresenta também, proporcionalmente, as menores quantidades de médicos, leitos de UTI e ventiladores, o que, aliado ao contexto da COVID-19 na região, com três de seus estados com maior incidência da doença, deixa seus habitantes mais suscetíveis a 
ficarem desassistidos e com possibilidade de danos irreversíveis, pela sobrecarga do SUS. Assim, para que não prevaleça a desigualdade no âmbito da saúde no enfrentamento à pandemia, é necessária a realocação de recursos que assegure a tomada de medidas preventivas, assim como esforço ativo para aparelhar, humana e fisicamente, os estabelecimentos de saúde da região norte do Brasil.

\section{REFERÊNCIAS}

1. European Respiratory Society (EU). The Global Impact of Respiratory Disease. Forum of International Respiratory Societies. Sheffield, England: European Respiratory Society; 2017.

2. World Health Organization. Global surveillance, prevention and control of chronic respiratory diseases: a comprehensive approach 2007. World Health Organization; 2007.

3. World Health Organization. Global Health Estimates 2016: Top 10 global causes of deaths, 2016. Geneva, Switzerland: World Health Organization; 2018.

4. Corrêa RA, São José BP, Malta DC, Passos VMA, França EB, Teixeira RA et al. Carga de doença por infecções do trato respiratório inferior no Brasil, 1990 a 2015: estimativas do estudo Global Burden of Disease 2015. Rev bras epidemiol. 2017; 20(Supl 1):171181.

5. Chen A, Jacobsen KH, Deshmukh AA, Cantor SB. The evolution of the disability-adjusted life year (DALY). Socio-econ plan sci. 2015; (49):10-15.

6. Instituto Brasileiro de Geografia e Estatística (BR). Estudos e PesquisasInformação Demográfica e Socioeconômica. Síntese de Indicadores Sociais: uma análise das condições de vida da população brasileira. Rio de Janeiro: IBGE; 2018.

7. Ministério da Saúde (BR). Secretaria de Vigilância em Saúde. Departamento de Análise de Saúde e Vigilância de Doenças Não Transmissíveis. Painel de monitoramento das principais causas de morte, segundo método Global Burden Disease/Brasil. Brasília: Ministério da Saúde; 2017.

8. Secretaria Estaduais de Saúde (SES). Casos confirmados, letalidade e óbitos por COVID-19 no Brasil. Brasília: SES; 2020.

9. Secretaria de Estado de Saúde do Amazonas (SUSAM). Cidades com maior incidência de casos de COVID- 
19. Número de casos por 100mil habitantes. Manaus; SUSAM, 2020.

10. Instituto Brasileiro de Geografia e Estatística (BR). Cidades e Estados. Rio de Janeiro: IBGE; 2018.

11. Instituto Brasileiro de Geografia e Estatística (BR). Estimativas da População. Rio de Janeiro: IBGE; 2019.

12. Instituto Brasileiro de Geografia e Estatística (BR). Censo Demográfico. Rio de Janeiro: IBGE; 2010.

13. Ministério da Saúde (BR). DATASUS: Informações de Saúde, Epidemiológicas e Morbidade: banco de dados [Internet]. Brasília: Ministério da Saúde; 2020 [acesso 2020 Abr 20]. Disponível em: http: / / tabnet.datasus.gov.br/cgi/de ftohtm.exe?sih/cnv/niuf.def.

14. Ministério da Saúde (BR). DATASUS: Informações de Saúde, Rede Assistencial: banco de dados [internet]. Brasília: Ministério da Saúde; 2020 [acesso 2020 Abr 20]. Disponível em: http: / /tabnet.datasus.gov.br/cgi/de ftohtm.exe?cnes/cnv/estabbr.def.

15. Ministério da Saúde (BR). Centro de Operações de Emergência em Saúde Pública | Doença pelo Coronavírus 2019 (COE-COVID19). Boletim 13 [internet]. Brasília: Ministério da
Saúde; 2020 [acesso 2020 Abr 28]. Disponível em: https: //portalarquivos.saude.gov.br /images/pdf/2020/April/21/BE13--Boletim-do-COE.pdf

16. Ministério da Saúde (BR). Resolução $\mathrm{n}^{\circ} 466$, de 12 de dezembro de 2012Diretrizes e normas regulamentadoras de pesquisa envolvendo seres humanos [Internet]. Diário Oficial da União, 2012 [acesso 2020 Mai 05]. Disponível

em:https://bvsms.saude.gov.br/bvs/ saudelegis/cns/2013/res0466_12_12 _2012.html

17. Ministério da Saúde (BR). Painel de casos de doença pelo coronavírus 2019 (COVID-19) no Brasil pelo Ministério da Saúde. Acumulado de casos e óbitos de COVID-19 por data de confirmação [Internet]. Brasília: Ministério da Saúde; 2020 [acesso 2020 Mai 01]. Disponível em: https: //covid.saude.gov.br/

18. Ministério da Saúde (BR). Secretaria de Atenção Especializada à Saúde. Departamento de Atenção Hospitalar, Domiciliar e de Urgência. Protocolo de Manejo Clínico da COVID-19 na Atenção Especializada [Internet]. Brasília: Ministério da Saúde; 2020. Disponível em: 
https://portalarquivos.saude.gov.br /images/pdf/2020/April/14/Protocol o-de-Manejo-Cl--nico-para-o-Covid19.pdf

19. Wu C, Chen X, Cai Y, Xia J, Zhou X, $\mathrm{Xu} \mathrm{S}$, et al. Risk Factors Associated With Acute Respiratory Distress Syndrome and Death in Patients With Coronavirus Disease 2019 Pneumonia in Wuhan, China. JAMA Intern Med. 2020.

20. Garnelo L, Sousa ABL, Silva CL. Regionalização em Saúde no Amazonas: avanços e desafios. Ciênc saúde coletiva. 2017; 22(4):12251234.

21. Teixeira C. Os princípios do sistema único de saúde. Texto de apoio elaborado para subsidiar o debate nas Conferências Municipal e Estadual de Saúde [Internet], Salvador; 2011; 1-10 [acesso 2020 Abr 24]. Disponível em: https://www.almg.gov.br/export/sit es/default/acompanhe/eventos/hots ites/2016/encontro_internacional_sa ude/documentos/textos_referencia/ 07_principios_sistema_unico_saude.p df

22. Carvalho L, Nassif LP, Lima LX. COVID-19 e Desigualdade no Brasil. 2020; 1-3. doi: 10.13140/RG.2.2.27014.73282.

23. Lloyd-Sherlock P, Ebrahim S, Geffen $L$, Mckee M. Bearing the brunt of COVID-19: older people in low and middle income countries. BMJ. 2020; 368:m1052.

24. Dias FLT, Mendonça FD, Pinto GM, Borges ISC, Oliveira SV. Doenças respiratórias no Triângulo Mineiro: Análise epidemiológica e projetiva com a pandemia de COVID-19. J Health Biol Sci. 2020; 8(1):1-6.

25. Fang $Y$, Nie $Y$, Penny $M$. Transmission dynamics of the COVID-19 outbreak and effectiveness of government interventions: A data-driven analysis. J Med Virol. 2020.

Conflito de interesses: Os autores declaram não haver conflito de interesses.

Participação dos autores:

- Concepção: Mendonça FD, Rocha SS, Pinheiro DLP, Oliveira SV.

- Desenvolvimento: Mendonça FD, Rocha SS, Pinheiro DLP, Oliveira SV.

- Redação e revisão: Mendonça FD, Rocha SS, Pinheiro DLP, Oliveira SV. 
Como citar este artigo: Mendonça FD, Rocha SS, Pinheiro DLP, Oliveira SV. Região Norte do Brasil e a pandemia de COVID-19: análise socioeconômica e epidemiológica. J Health NPEPS. 2020; 5(1):20-37.

Submissão (Fast Track COVID-19): 05/05/2020

Aceito (Fast Track COVID-19): 09/05/2020

Publicado (Fast Track COVID-19): 10/05/2020 